\title{
Evaluation of Plasma MicroRNAs as Diagnostic and Prognostic Biomarkers in Pancreatic Adenocarcinoma: miR-196a and miR-210 Could Be Negative and Positive Prognostic Markers, Respectively
}

\author{
Qi Yu, ${ }^{1}$ Changqing Xu, ${ }^{2}$ Wei Yuan, ${ }^{3}$ Chengfeng Wang, ${ }^{4}$ Ping Zhao, \\ Lianzhen Chen, ${ }^{5}$ and Jie $\mathrm{Ma}^{3}$ \\ ${ }^{1}$ Department of General Surgery, Beijing Rehabilitation Hospital, Capital Medical University, Beijing 100044, China \\ ${ }^{2}$ Department of Digestive Medicine, Qianfoshan Hospital, Shandong University, 16766 Jingshi Road, Jinan 250014, China \\ ${ }^{3}$ State Key Laboratory of Molecular Oncology, National Cancer Center/Cancer Hospital, \\ Chinese Academy of Medical Sciences and Peking Union Medical College, Beijing 100021, China \\ ${ }^{4}$ Department of Abdominal Surgery, Cancer Hospital of Chinese Academy of Medical Sciences, \\ Peking Union Medical College, Beijing, China \\ ${ }^{5}$ Beijing Hospital, National Center of Gerontology, Beijing 100730, China
}

Correspondence should be addressed to Ping Zhao; dr.zhaoping@263.net and Lianzhen Chen; yjkclz@163.com

Received 22 November 2016; Accepted 9 January 2017; Published 29 March 2017

Academic Editor: Xin-yuan Guan

Copyright (C) 2017 Qi Yu et al. This is an open access article distributed under the Creative Commons Attribution License, which permits unrestricted use, distribution, and reproduction in any medium, provided the original work is properly cited.

\begin{abstract}
Background. Identifying diagnostic and prognostic biomarkers that could be targeted in the therapy of pancreatic cancer is essential. Objective. Investigations were conducted with respect to plasma miRNA (miR-21, miR-210, miR-155, miR-196a, miR-20a, and miR25) expression and clinicopathologic factors to evaluate the prognostic value of miRNAs in pancreatic ductal adenocarcinoma (PDAC). Methods. Plasma miRNAs were detected by real-time quantitative PCR, and the association with clinicopathologic factors was subsequently performed by univariate and multivariate analyses. Results. Six miRNAs expressed significantly higher in PDAC patients than in normal individuals were identified. Receiver operating characteristic (ROC) curves were constructed. It was evident that miRNA expression associated with PDAC, lymph node metastasis, serosal infiltration, and comprehensive therapy reached significance for overall survival. High miR-196a expression was associated with poor survival $(P=0.001)$, whereas high miR-210 expression was significantly associated with improved survival $(P=0.003)$. Multivariate survival analysis indicated that the miR-210 and miR-196a expression signature, lymph node metastasis, and comprehensive therapy were independent factors affecting overall survival. Conclusions. MiRNA expression profile is distinctive in PDAC. Aberrant expression of certain miRNAs was remarkably involved in shaping the overall survival time, which include miR-196a overexpression and decreased miR-210 expression.
\end{abstract}

\section{Introduction}

Pancreatic cancer is one of the most fatal cancers worldwide, and it has been a therapeutic challenge for years, due to difficulties and delays in diagnosis, which results in poor prognosis. Pancreatic cancer ranks the fourth leading cause of cancer-related deaths in America [1] and the sixth in China [2]. Poor diagnosis results due to the relatively special anatomical position of the pancreas, which is situated deeply and adjacent to the superior mesenteric vein and artery.
Also, the patients in the early stages of pancreatic cancer do not present specific symptoms. Moreover, the radical resection rate of this malignancy is only $10 \%-15 \%$ [3]. Despite surgical resection, the median overall survival in patients with advanced disease stage is about 6 months [4].

During the last decade, the prognosis of pancreatic cancer has not showed any remarkable improvements regardless of the progress in surgical techniques and diagnostic methods. Hence, it is vital to identify distinct in vivo markers for pancreatic cancer in terms of diagnosis and prognosis. This 
may help to improve the success rates of radical resection and assist in decisions during therapeutic protocols.

The aberrant expression of several miRNAs, considered as diagnostic markers of malignancies, has been widely reported. The overexpression and underexpression of miRNAs have been detected in resected pancreatic cancer tissue specimens, some of which have been evaluated as prognostic markers [5-8]. It was revealed that miRNAs were stable in the plasma and showed the potential to serve as diagnostic and/or prognostic biomarkers. Earlier report about plasma miRNA profile in patients with pancreatic cancer, by Wang et al. [9], indicated that four miRNAs (miR-21, miR-210, miR155, and miR-196a) were found to be highly expressed in pancreatic cancer tissues, as well as in the plasma. The four miRNAs had a sensitivity of $64 \%$ and a specificity of $89 \%$ for pancreatic cancer. Subsequently, however, Liu et al. [10] reported 6 new miRNA-based biomarkers (miR-20a, miR24, miR-25, miR-99a, miR-185, and miR-191) along with miR21 and indicated that they were highly sensitive and specific in distinguishing various stages of PDAC from cancer-free pancreatic tissues. In addition, miR-21 expression levels in the serum were substantially associated with overall survival. On analyzing the diagnostic value of miR-21, miR-155, and miR196a, Kong et al. [11] suggested that serum miR-196a could be a potential marker for PDAC prognosis.

Considering that as most studies used tissues resected from patients undergoing surgery, we evaluate the association between the plasma miRNA expression profiles in the resected tissues with the overall survival of PDAC patients. This may help in the decision-making process of the therapeutic method in PDAC patients. In this study, six known miRNAs (miR-21, miR-210, miR-155, miR-196a, miR-20a, and miR-25) whose diagnostic value was previously reported were selected for further examination of their prognostic values in PDAC.

\section{Materials and Methods}

2.1. Experimental Subjects. Our study recruited 31 PDAC patients from the Department of abdominal surgery of the Cancer Hospital and Institute of the Chinese Academy of Medical Sciences (Beijing, China) between September 2012 and September 2013, who were confirmed by either pathological examination or fine-needle aspiration cytology (during surgery or by EUS). Twenty-eight healthy individuals were taken up in the study as control, who are all confirmed with healthy conditions after undergoing blood tests and imaging examinations in the Department of Cancer Prevention. This study was conducted in conformity to The Code of Ethics of the World Medical Association (Declaration of Helsinki), printed in the British Medical Journal (18 July, 1964).

Among the 31 PDAC patients, 20 (64.5\%) were male and $11(35.5 \%)$ were female, and the age ranged from 47 to $73(48.82 \pm 9.75)$ years. The tumor in 26 patients $(83.9 \%)$ was in the head and neck region of the pancreas and in 5 patients (16.1\%) in the pancreas body and tail. Nine patients (29.1\%) had radical surgery, achieving R0 resection. Seventeen patients $(54.8 \%)$ received palliative surgery. The remaining 5 patients $(16.1 \%)$ did not undergo any interventions for tumor metastasis or for other reasons. Comprehensive therapy includes postoperative chemotherapy, radiotherapy, chemoradiotherapy, or intraoperative radiotherapy. Distant liver, lung, or mesenteric dissemination, peritoneal seeding, and supraclavicular lymph node metastases were evaluated before the surgery.

For the patients receiving radical surgery, several characteristics which include tumor size, lymph node metastasis, and serosal or vessel infiltration were evaluated based on the pathological examination. In the patients who received palliative surgery and in those who were unsuitable for surgical treatment, these characteristics were assessed by endoscopic ultrasonography, dynamic computed tomography, or magnetic resonance imaging.

Demographic data for PDAC patients and healthy controls are listed in Table 1. No significant differences in terms of gender, age, cigarette smoking, and alcohol consumption between the two groups $(P>0.05)$ were observed. Clinical characteristics of the PDAC patients are detailed in Table 3.

2.2. Plasma Collection. Blood samples were collected from individuals in the two groups before surgery or other therapies and centrifuged at $1,200 \mathrm{~g}$ at $4^{\circ} \mathrm{C}$ for $10 \mathrm{~min}$ to obtain the plasma for miRNA detection. The plasma was then further centrifuged at $1,2000 \mathrm{~g}$ at $4^{\circ} \mathrm{C}$ for $10 \mathrm{~min}$ to eliminate larger molecules. Each plasma sample $(200 \mu \mathrm{L}$ at least) was transferred into $1.5 \mathrm{~mL}$ Eppendorf tubes and stored at $-80^{\circ} \mathrm{C}$ for further total RNA isolation.

2.3. RNA Extraction. Plasma samples were thawed on ice and $200 \mu \mathrm{L}$ was transferred into a tube containing $1000 \mu \mathrm{L}$ Qiazol lysis reagent (Qiagen, Germany). After vortexing, the tube containing the lysate was left at room temperature $\left(15-25^{\circ} \mathrm{C}\right)$ for $5 \mathrm{~min}$. Subsequently, $3.5 \mu \mathrm{L}$ miRNeasy Serum/Plasma Spike-In Control $\left(1.6 \times 10^{8}\right.$ copies $/ \mu \mathrm{L}$ working solution, miR39) (Qiagen, Germany) was added to the tube and samples were mixed thoroughly. RNA was isolated by using miRNeasy Serum/Plasma Kit (Qiagen, Germany) following the manufacture's protocol. Each $12 \mu \mathrm{L}$ RNA pellet was placed in the RNase-free collection tube and stored at $-80^{\circ} \mathrm{C}$.

2.4. Absolute Quantification of Plasma miRNA. Briefly, miRNeasy Serum/Plasma Spike-In Control (miR-39) and $2 \mu \mathrm{L}$ of plasma-derived total RNA were reversely transcribed according to the Reverse Transcription Reaction Kit protocol. cDNA from miR-39 was diluted to different concentrations following the cDNA serial dilution protocol. All cDNAs were prepared for real-time PCR according to miScript SYBR Green PCR Kit (Qiagen, Germany) protocol. The mean CT values of miRNeasy Serum/Plasma Spike-In Control (miR39) and samples from each reaction were calculated by using the Roche ${ }^{\circledR}$ LightCycler ${ }^{\circledR} 480$ software. The standard curves were generated by plotting the log copy number and $\mathrm{CT}$ values. The recovery was determined based on absolute quantification (copies/ $\mu \mathrm{L}$ ) for each sample.

2.5. Statistical Analysis. To employ the SPSS 17.0 software, the Chi-square test was used to examine the differences in the demographic data between the two groups. Quantification 
TABLE 1: Demographic data of human pancreatic ductal adenocarcinoma patients $(\mathrm{PaC})$ and healthy control individuals.

\begin{tabular}{|c|c|c|c|}
\hline Variable & $\mathrm{PaC}$ & Control & $P$ value \\
\hline \multicolumn{4}{|l|}{ Gender } \\
\hline Male & 20 & 18 & 0.985 \\
\hline Female & 11 & 10 & \\
\hline Age (years) & $48.82 \pm 9.75$ & $44.65 \pm 6.85$ & 0.155 \\
\hline \multicolumn{4}{|l|}{ Cigarette smoking } \\
\hline Yes & 10 & 11 & 0.713 \\
\hline No & 21 & 28 & \\
\hline Alcohol consumption & & & 0.306 \\
\hline Yes & 14 & 9 & \\
\hline No & 17 & 19 & \\
\hline
\end{tabular}

TABLE 2: Differential expression of six miRNAs in the plasma of the PDAC and the control groups.

\begin{tabular}{lccc}
\hline & PDAC $(\lg$ copies $/ \mu \mathrm{L})$ & Control $(\lg$ copies $/ \mu \mathrm{L})$ & $P$ value \\
\hline miR-21 & $6.34 \pm 0.44$ & $5.65 \pm 0.69$ & 0.000 \\
miR-210 & $4.94 \pm 0.43$ & $4.70 \pm 0.39$ & 0.013 \\
miR-155 & $3.76 \pm 0.74$ & $3.26 \pm 0.30$ & 0.002 \\
miR-196a & $3.47 \pm 0.44$ & $2.82 \pm 0.75$ & 0.000 \\
miR-20a & $6.79 \pm 0.44$ & $5.50 \pm 0.53$ & 0.000 \\
miR-25 & $6.61 \pm 0.37$ & $6.26 \pm 0.33$ & 0.000 \\
\hline
\end{tabular}

of miRNAs' expression in the two groups was compared by Student's $t$-test (SPSS 17.0 software). Receiver operating characteristic curves (ROC curves) were established to further confirm miRNA expression in PDAC diagnosis. For the univariate survival analysis of conventional prognostic factors, the analysis of median survival time of postoperative survival between the two groups was evaluated based on clinicopathological or miRNAs expression' level in terms of the Kaplan-Meier method and log-rank analysis. Variables with significant $P$ values from the univariate analysis were taken into the multivariate analysis by using Cox regression. Only $P$ value of less than 0.05 (two-sided) was defined as statistically significant.

\section{Results}

3.1. $m i R-21, m i R-210, m i R-155, m i R-196 a, m i R-20 a$, and $m i R-$ 25 Have Strong Diagnostic Value of PDAC Patients. The expressions of plasma miRNA (miR-21, miR-210, miR-155, miR-196a, miR-20a, and miR-25) in PDAC patients and healthy controls were detected and compared. The logarithm (base 10) of expression was considered as the evaluation level for its normal distribution. As shown in Table 2 and Figure 1, the expression of six miRNAs in PDAC patients was significantly higher than that in healthy individuals. ROC curves were illustrated in Figure 2. The AUC (area under the curve) was 0.845 for miR-21 [95\% CI, 0.740-0.949], 0.687 for miR-210 (95\% CI, 0.543-0.831), 0.822 for $\mathrm{miR}-155(95 \%$ CI, 0.707-0.937), 0.791 for miR-196a (95\% CI, 0.665-0.916), 0.884 for miR-20a (95\% CI, $0.790-0.978$ ), and 0.763 for miR25 (95\% CI, 0.635-0.891), respectively.

3.2. $m i R-196 a$ and miR-210 Expression Reached Significance in Univariate Analysis for Survival Time of PDAC Patients. Prognoses were performed in December 2013, obtaining available data from 31 cases by telephonic follow-up. The overall median survival time was 7.1 months, ranging from 2.3 to 15.2 months. Kaplan-Meier survival curves were constructed and compared by the log-rank test. Factors such as surgical method, lymph node metastasis, serosal infiltration, and comprehensive therapy were largely involved in the survival of the patient (Table 2). Based on the logarithm of absolute quantitative miRNA expression, the median values were taken as a cut-off to catalogue patients into high- and low-miRNA groups, respectively. The median survival times of the two groups for each miRNA were compared. A median survival of 6.3 months (95\% CI, 2.3-10.3) was obtained in the high miR-196a group which was significantly shorter than that of 12.5 months (95\% CI, 10.0-15.0) in the low miR-196a group $(P=0.001)$. Furthermore, high miR-210 expression with a median survival of 11.7 months (95\% CI, 6.8-16.5) was considerably correlated with an improved survival in comparison to the low miR-210 expression with a median survival of 6.6 months $(95 \% \mathrm{CI}, 5.3-7.9)(P=0.003)$. There were no significant differences in terms of the survival times $(P=0.714,0.822,0.394$, and 0.362 , resp. $)$ between the high- and low-expression groups for the other four miRNAs (miR-21, miR-155, miR-20a, and miR-25) (Tables 3 and 4, Figure 3).

3.3. miR-210 Expression, miR-196a Expression, Lymph Node Metastasis, and Comprehensive Therapy Were Independent Factors for Survival Time of PDAC Patients by Multivariate Analysis. By Cox regression analysis, multivariate survival analysis was investigated for all 6 variables with significant $P$ values in univariate analysis, such as surgical method, lymph node metastasis, serosal infiltration, comprehensive therapy, and expression of miR-196a and miR-210. Table 5 showed 
TABLE 3: Univariate survival analysis of conventional prognostic factors based on the characteristics of the PDAC patients corresponding to the survival time.

\begin{tabular}{|c|c|c|c|}
\hline Characteristic & Number & Median survival (month) & $P$ value \\
\hline Gender & & & 0.550 \\
\hline Male & 20 & 7.0 & \\
\hline Female & 11 & 8.7 & \\
\hline Age (years) & & & 0.521 \\
\hline$\geq 60$ & 17 & 10.7 & \\
\hline$<60$ & 14 & 6.9 & \\
\hline Cigarette smoking & & & 0.255 \\
\hline Yes & 10 & 6.5 & \\
\hline No & 21 & 7.5 & \\
\hline Alcohol consumption & & & 0.154 \\
\hline Yes & 10 & 7.0 & \\
\hline No & 21 & 8.1 & \\
\hline Abdominal pain & & & 0.341 \\
\hline Yes & 13 & 6.8 & \\
\hline No & 18 & 8.1 & \\
\hline Jaundice & & & 0.058 \\
\hline Yes & 9 & 6.9 & \\
\hline No & 22 & 9.9 & \\
\hline Tumor location & & & 0.531 \\
\hline Head and neck & 26 & 6.1 & \\
\hline Body and tail & 5 & 8.8 & \\
\hline Operation method & & & 0.009 \\
\hline Radical surgery & 9 & 11.2 & \\
\hline Palliative surgery & 17 & 6.3 & \\
\hline No surgery & 5 & 5.5 & \\
\hline Tumor size $(\mathrm{cm})$ & & & 0.289 \\
\hline$\leq 2$ & 4 & 9.3 & \\
\hline$>2-\leq 4$ & 18 & 10.1 & \\
\hline$>4$ & 9 & 6.7 & \\
\hline Lymph node metastasis & & & 0.001 \\
\hline Yes & 19 & 5.0 & \\
\hline No & 12 & 12.5 & \\
\hline Serosal infiltration & & & 0.002 \\
\hline Yes & 20 & 7.0 & \\
\hline No & 11 & 13.2 & \\
\hline Vessel infiltration & & & 0.053 \\
\hline Yes & 17 & 6.8 & \\
\hline No & 14 & 9.5 & \\
\hline Comprehensive therapy & & & 0.024 \\
\hline Yes & 19 & 10.4 & \\
\hline No & 12 & 6.9 & \\
\hline Distant metastasis & & & 0.079 \\
\hline Yes & 6 & 6.8 & \\
\hline No & 25 & 8.1 & \\
\hline
\end{tabular}

that the overall survival time was significantly dependent on miR-210 expression, miR-196a expression, lymph node metastasis, and comprehensive therapy $(P=0.021,0.013$, 0.009 , and 0.045 , resp.). Therefore, miR-196a and miR210 could be regarded as negative and positive prognostic markers, respectively.

\section{Discussion}

Facing the serious therapeutic and prognostic considerations of pancreatic adenocarcinoma, it is necessary to thoroughly investigate the expression of plasma microRNAs so as to establish their diagnostic and prognostic value for this 


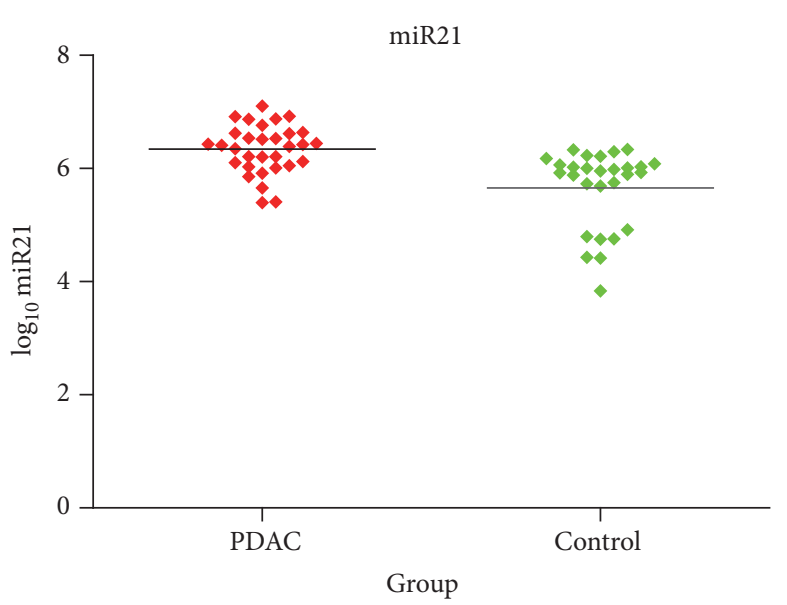

(a)

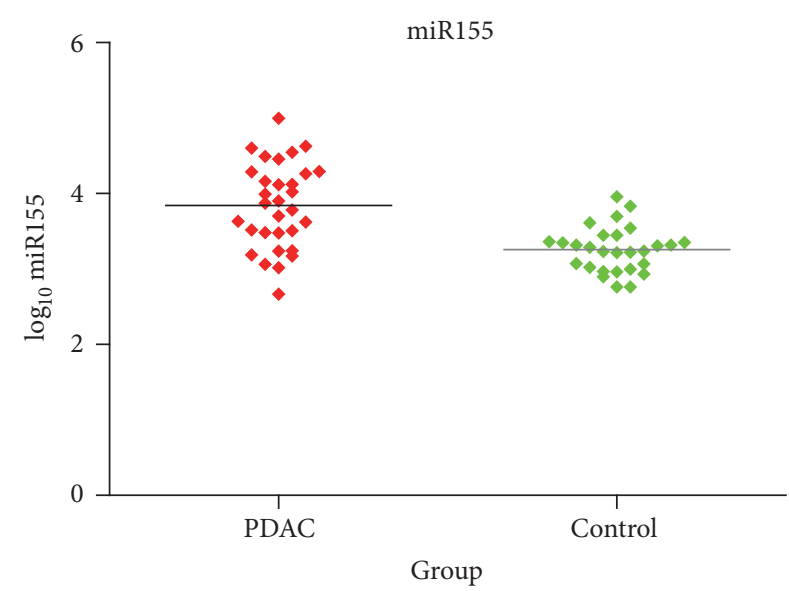

(c)

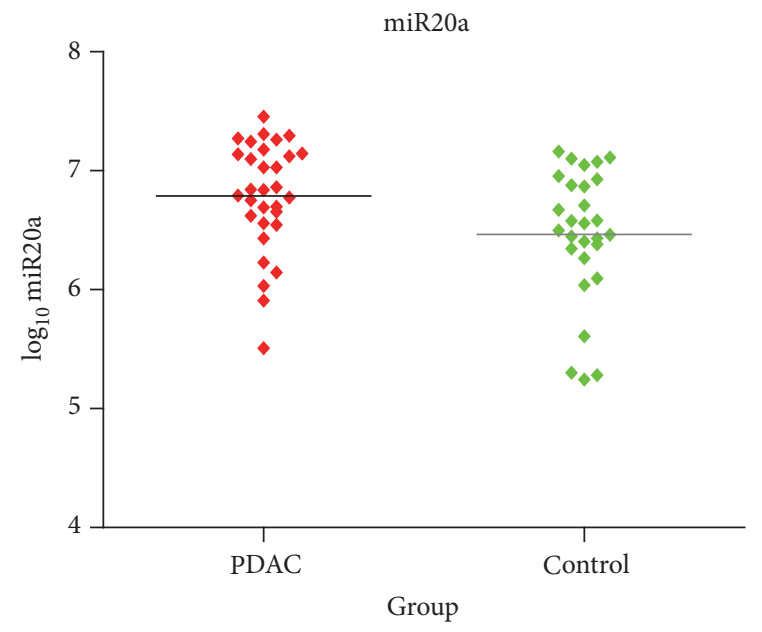

(e)

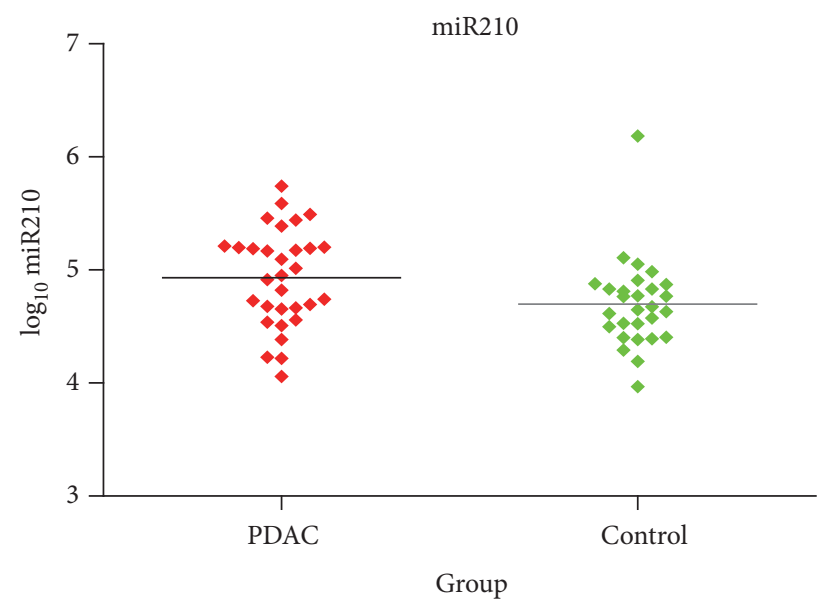

(b)

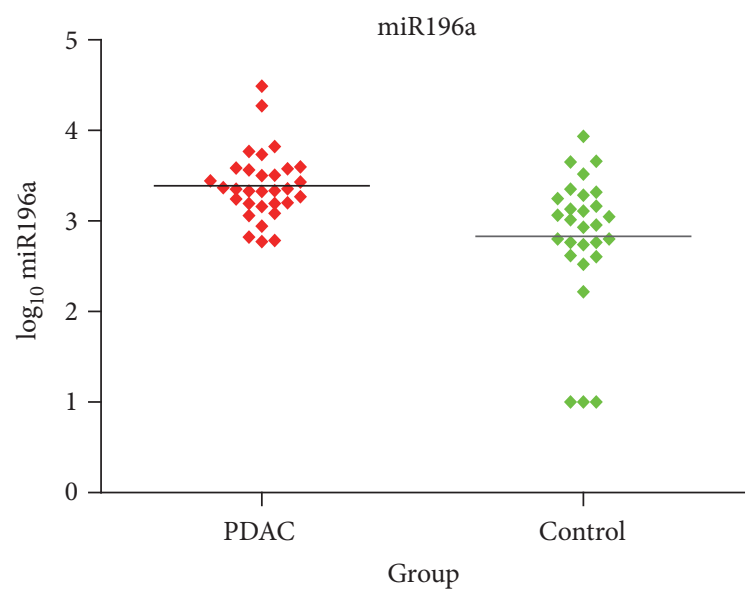

(d)

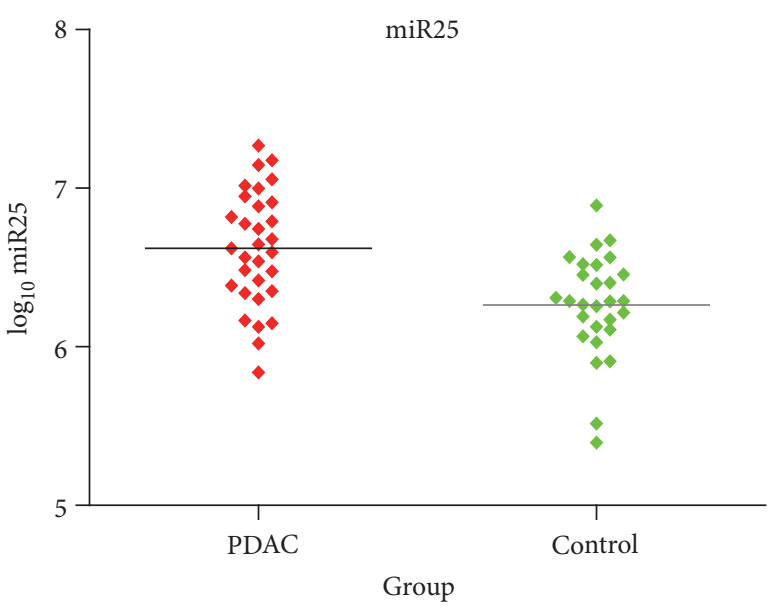

(f)

FIGURE 1: Expression levels for the six miRNAs in the plasma from PDAC patients and control individuals. (a)-(f) represent miR-21, miR-210, miR-155, miR-196a, miR-20a, and miR-25.

condition. In this study, we have further confirmed the diagnostic value of six plasma miRNAs (miR-21, miR-210, miR-155, miR-196a, miR-20a, and miR-25), all of which were expressed at significantly higher levels in PDAC patients than in controls, with the $P$ values in the range of 0.000 to 0.013 and the AUDs of $0.845,0.687,0.822,0.791,0.884$, and 0.763 , respectively. More importantly, we convincingly showed that plasma miR-196a overexpression was significantly associated 
TABLE 4: Univariate survival analysis of prognostic factors based on miRNA expression.

\begin{tabular}{lccc}
\hline & MST of lower expression (month) & MST of higher expression (month) & $P$ value \\
\hline miR-21 & 7.0 & 7.4 & 0.714 \\
miR-210 & 6.6 & 11.7 & 0.003 \\
miR-155 & 7.0 & 7.1 & 0.822 \\
miR-196a & 12.5 & 6.3 & 0.001 \\
miR-20a & 11.7 & 7.0 & 0.394 \\
miR-25 & 6.3 & 10.1 & 0.362 \\
\hline
\end{tabular}

TABLE 5: Multivariate analysis of survival time of patients with PDAC.

\begin{tabular}{lcccr}
\hline Covariant & $B$ coefficient & SE & $P$ value & $95 \%$ confidence interval \\
\hline Lymph node metastasis & -0.198 & 0.231 & 0.009 & $0.098-1.212$ \\
Comprehensive therapy & 1.211 & 0.545 & 0.045 & $0.055-2.051$ \\
miR-210 & 0.545 & 0.594 & 0.021 & $0.633-1.098$ \\
miR-196a & -1.255 & 0.051 & 0.013 & $0.923-1.055$ \\
\hline
\end{tabular}

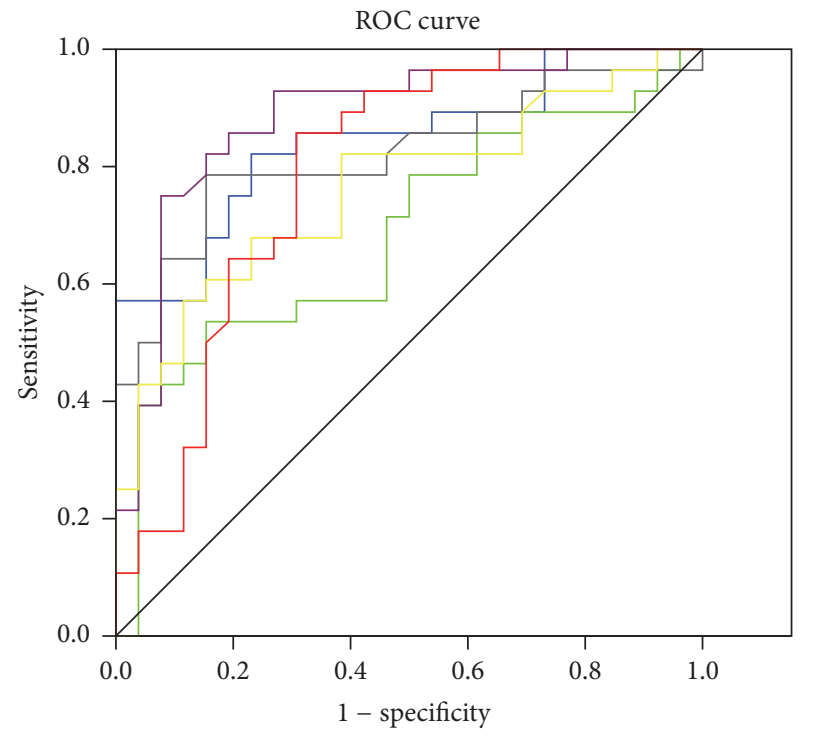

Source of the curve

$\begin{array}{ll}-\mathrm{m} 21 & \mathrm{~m} 25 \\ \mathrm{~m} 210 & -\mathrm{m} 196 \mathrm{a} \\ \mathrm{m} 155 & - \text { Reference line } \\ \mathrm{m} 20 \mathrm{a} & \end{array}$

Figure 2: The ROC curves for the expression of the six plasma miRNAs and the corresponding calculated AUCs.

with poorer survival rate. On the contrary, plasma miR210 overexpression was observed to demonstrate significantly prolonged survival in PDAC patients. All these indications were based on the multivariate analysis with confounding clinical factors.

In addition to pancreatic cancer, the overexpression of miR-21 has been detected and correlated with poor survival in numerous cancers, such as glioma [12], breast cancer [13], colorectal cancer [14], gastric cancer [15], and prostate cancer $[10,16]$. Thus overexpression of such miRNA may lack specificity and sensitivity. MiR-21 has been believed to function as an oncogenic miRNA. Some of miR-21-targeted genes or proteins have been investigated in previous studies. MiR-21 has been shown to affect cell proliferation, invasion, and the clinical outcome by downregulating the expression of tumor suppressors, such as PDCD4 [17, 18], TIMP3 [18], RECK [19], and KRIT1 [20]. The targeting of some other proteins, including Bcl-2, maspin, and PTEN, was also confirmed $[6,21]$. Besides, overexpression of miR-21 could be involved in the inhibition of apoptosis as well as the acquisition of invasive properties [22-24]. MiR-155 has been reported to be an important diagnostic biomarker for PDAC as it could perform its oncogenic function by repressing proapoptotic tumor proteins [25] and regulate the invasion and migration of pancreatic cancer cells by modulating the STAT3 signaling pathway and by reducing SOCS1 expression [26]. Furthermore, the expression of MLH1 [27] and tumor suppressor SEL1L [28] appeared to be miR-155-modulating targets for the oncogenic function. Additionally, miR-155 was observed to indicate early pancreatic neoplasia $[29,30]$. As for the prognosis, some studies demonstrated that high expression of miR-155 in tissues was correlated with poor survival [5-7]. However, the prognostic value of plasma miR-21 and miR-155 was not found in our study. This was probably explained as a result of the differences between the tissue and the plasma miRNAs expression levels. Alternatively, the limited sample size in this study may also be a reason.

High expression of miR-20a (the miR-17-92 cluster) has been reported in the plasma and tissue samples of patients with colon [31, 32], gastric [33, 34], prostate [35], and cervical cancer $[36,37]$. In addition, miR-20a was upregulated at the different stages during nasopharyngeal carcinoma (NPC) progression. The expression level of such miRNA was reversibly associated with the prognosis of NPC [38]. The upregulation of miR-20a was suggested to enhance the metastatic potential of osteosarcoma [39] and breast cancer cells [40]. Interestingly, miR-20a was found to act as a tumor suppressor in breast cancer $[41,42]$ and inhibit the proliferation and invasion of pancreatic cells by negatively regulating 


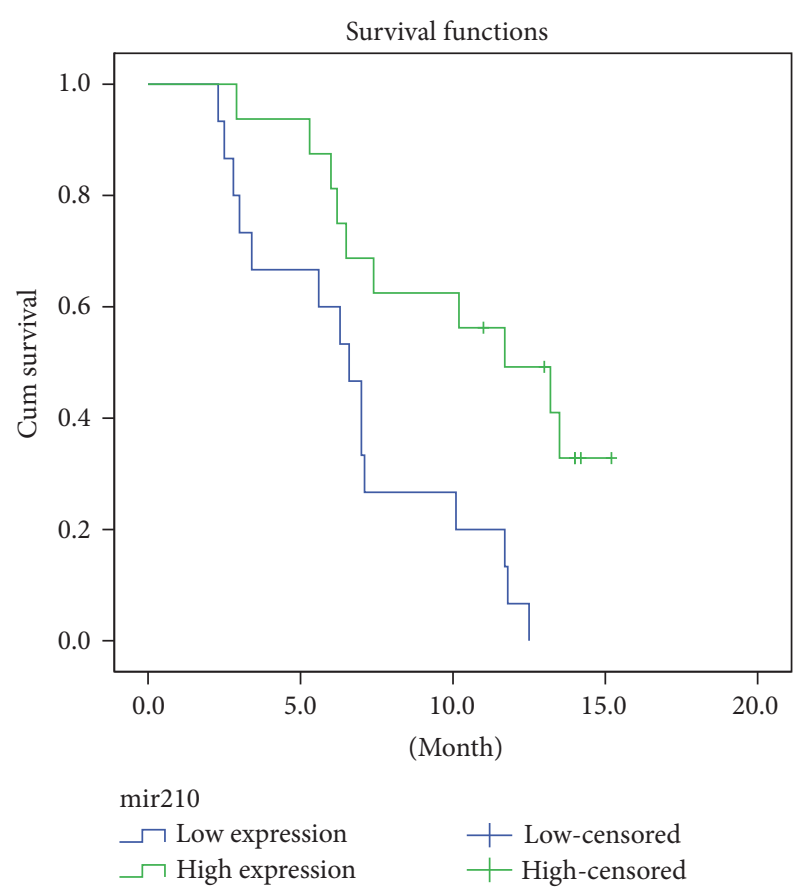

(a)

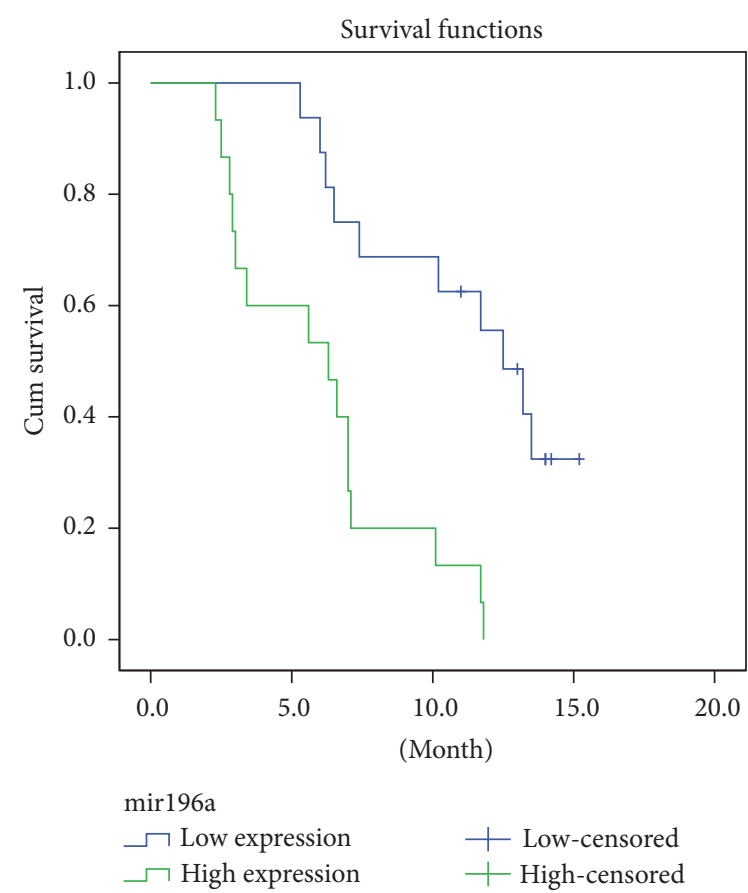

(b)

Figure 3: Kaplan-Meier overall survival curves for PDAC patients based on expression levels of the plasma miRNAs. The median logarithm value of the relative expression levels was used as a cut-off to classify patients into high- and low-expression miRNA groups. (a) and (b) stand for Kaplan-Meier overall survival curves for miR-210 and miR-196a, respectively.

STAT-3 protein expression [43] due to its possible doublesided role in tumors. MiR-25 (the miR-106b-25 cluster) is another double-sided miRNA in tumors. It could accelerate tumor growth and apoptosis by targeting Bim in gastric [44] and liver cancers [45]. Also, it appeared to promote ESCC cell migration and invasion both by overexpressing in tissues and by suppressing DH1 expression [46]. On the other hand, downregulated miR-25 was found in human colon cancer tissues, in which it inhibited cell proliferation and migration in cell lines and suppressed the growth of colon cancer cell xenografts by targeting Smad7 in colon cancer [47]. Therefore, we speculated that the genetic function of miR-20a and miR-25 may be tissue-specific. To date, the roles of miR-20a and miR-25 in the prognosis of pancreatic cancer have not been reported. In this study, there were no evidence to ascertain the expression of miR-20a and miR-25 with respect to survival time in PDAC patients. Thus, more in-depth studies need to be carried out in order to confirm their prognostic potential in PDAC.

Some previous studies evaluated tissue or plasma miR196a during the survival of PDAC patients $[48,49]$. Our study further confirmed that high expression of plasma miR196a was an independent factor informative about survival time $(P=0.013)$. MiR-196a has been well believed to be associated with abnormal apoptosis, invasion, and proliferation of pancreatic cancer cells by downregulating ING5 expression [50]. In addition, miR-196a was also observed to be significantly deregulated in PanIN II-III, the early stage of PDAC [51]. A number of studies have also been conducted in the relationship between the expression of miR-196a and other malignancies $[52,53]$.

This is the first study, in the prognosis of PDAC patients, to demonstrate that plasma miR-210 was an independent positive factor based on multivariate analyses $(P=0.021)$. Despite the possible controversy caused by the contradiction between the diagnostic and prognostic value, our observation was consistent with studies regarding the diagnosis and prognosis of other malignancies such as non-small cell lung cancer [54], renal cell carcinoma [55], and soft-tissue sarcoma [56].

MiR-210 expression was indicated to regulate downstream genes, possibly by ERK- and PI3K/Akt-dependent signaling pathways [57] in the presence of a relative miR-210 upregulation in cardiomyocytes [58] and in adipose-derived stem cells [59]. Several studies found that miR-210 may function as a tumor suppressor by inhibiting ovarian cancer cell proliferation $[60,61]$. Disruption of iron homeostasis in cancer cells could be a possible mechanism by which miR210 constrained cancer cell proliferation [62]. Although miR210 expression can induce cell migration [57], it did not exhibit any changes in the proliferation of Panc- 1 cell lines [63]. MiR-210 was suggested to be able to regulate normoxic gene expression involved in tumor initiation and repress the initiation of tumor growth [64]. This might explain the mechanism of its positive impact on the prognosis of PDAC patients in this study. Greither et al. [7] reported that miR210 tissue expression reversely correlated with survival time in PDAC patients. The sample origin, however, was tissue, 
not plasma. Moreover, further validation was performed in a breast cancer cell line (MCF-7) or tissue, neck cancer tissue to confirm that miR-210 was an independent negative prognostic factor for breast cancer and neck cancer patients $[65,66]$. And also a meta-analysis of hazard ratio (HR) [67] was performed to evaluate the prognostic role of the miR-210 in different tumors including 9 published studies dealing with various carcinomas suggested that higher expression of miR210 correlated with worse survival time, especially in breast cancer, and all of the samples are from tissues and most of the samples are from breast cancer patients.

The different prognostic value of miR-210 may be illustrated by the hypoxic environment it exerts the function and miR-210 having different regulatory effects on different cancer type and sample origin, plasm, or tissue. Therefore, the overall balance between pro- and antitumorgenesis genes differs in the specific tumor. Taken together, intensive studies by using large sample sizes are needed to investigate the paired-expression of miRNAs in both the tissue and the plasma for a variety of malignancies.

In summary, our study provided corroborative evidence to support the diagnostic value of six plasma miRNAs (miR21, miR-210, miR-155, miR-196a, miR-20a, and miR-25) in PDAC patients. Significantly, miR-210 displayed a positive value in terms of prognosis and miR-196a was negative for prognosis. This observation may contribute to the clinical guidance. Furthermore, in-depth studies are needed to illuminate the molecular mechanisms and the therapeutic target(s) of miRNAs in PDAC patients.

\section{Competing Interests}

The authors declare that they have no competing interests.

\section{Acknowledgments}

This study was supported by the National Key Research and Development plan (Grant no. 2016YFA0201503), the National Basic Research Program of China (Grant no. 2014CB542103), and CAMS Innovation Fund for Medical Sciences (CIFMS) (Grant no. 2016-I2M-1-001).

\section{References}

[1] A. Jemal, R. Siegel, E. Ward et al., "Cancer statistics, 2008," CA Cancer Journal for Clinicians, vol. 58, no. 2, pp. 71-96, 2008.

[2] W. Chen, R. Zheng, S. Zhang et al., "The incidences and mortalities of major cancers in China, 2009," Chinese Journal of Cancer, vol. 32, no. 3, pp. 106-112, 2013.

[3] S. F. Sener, A. Fremgen, H. R. Menck, and D. P. Winchester, "Pancreatic cancer: a report of treatment and survival trends for 100,313 patients diagnosed from 1985-1995, using the national cancer database," Journal of the American College of Surgeons, vol. 189, no. 1, pp. 1-7, 1999.

[4] J. M. Winter, J. L. Cameron, K. A. Campbell et al., "1423 Pancreaticoduodenectomies for pancreatic cancer: a singleinstitution experience," Journal of Gastrointestinal Surgery, vol. 10, no. 9, pp. 1199-1211, 2006.
[5] I. G. Papaconstantinou, A. Manta, M. Gazouli et al., "Expression of micrornas in patients with pancreatic cancer and its prognostic significance," Pancreas, vol. 42, no. 1, pp. 67-71, 2013.

[6] N. B. Jamieson, D. C. Morran, J. P. Morton et al., "MicroRNA molecular profiles associated with diagnosis, clinicopathologic criteria, and overall survival in patients with resectable pancreatic ductal adenocarcinoma," Clinical Cancer Research, vol. 18, no. 2, pp. 534-545, 2012.

[7] T. Greither, L. F. Grochola, A. Udelnow, C. Lautenschläger, P. Würl, and H. Taubert, "Elevated expression of microRNAs 155, 203, 210 and 222 in pancreatic tumors is associated with poorer survival," International Journal of Cancer, vol. 126, no. 1, pp. 7380, 2010.

[8] E. Giovannetti, A. van der Velde, N. Funel et al., "Highthroughput microRNA (miRNAs) arrays unravel the prognostic role of MiR-211 in pancreatic cancer," PLOS ONE, vol. 7, no. 11, Article ID e49145, 2012.

[9] J. Wang, J. Chen, P. Chang et al., "MicroRNAs in plasma of pancreatic ductal adenocarcinoma patients as novel bloodbased biomarkers of disease," Cancer Prevention Research, vol. 2, no. 9, pp. 807-813, 2009.

[10] R. Liu, X. Chen, Y. Du et al., "Serum microRNA expression profile as a biomarker in the diagnosis and prognosis of pancreatic cancer," Clinical Chemistry, vol. 58, no. 3, pp. 610 $618,2012$.

[11] X. Kong, Y. Du, G. Wang et al., "Detection of differentially expressed microRNAs in serum of pancreatic ductal adenocarcinoma patients: MiR-196a could be a potential marker for poor prognosis," Digestive Diseases and Sciences, vol. 56, no. 2, pp. 602-609, 2011.

[12] Q. Wang, P. Li, A. Li et al., "Plasma specific miRNAs as predictive biomarkers for diagnosis and prognosis of glioma," Journal of Experimental and Clinical Cancer Research, vol. 31, no. 1, article 97, 2012.

[13] S. Kumar, R. Keerthana, A. Pazhanimuthu, and P. Perumal, "Overexpression of circulating miRNA-21 and miRNA-146a in plasma samples of breast cancer patients," Indian Journal of Biochemistry and Biophysics, vol. 50, no. 3, pp. 210-214, 2013.

[14] E. K. O. Ng, W. W. S. Chong, H. Jin et al., "Differential expression of microRNAs in plasma of patients with colorectal cancer: a potential marker for colorectal cancer screening," Gut, vol. 58, no. 10, pp. 1375-1381, 2009.

[15] J.-L. Wang, Y. Hu, X. Kong et al., "Candidate microRNA biomarkers in human gastric cancer: a systematic review and validation study," PLoS ONE, vol. 8, no. 9, Article ID e73683, 2013.

[16] P. S. Mitchell, R. K. Parkin, E. M. Kroh et al., "Circulating microRNAs as stable blood-based markers for cancer detection," Proceedings of the National Academy of Sciences of the United States of America, vol. 105, no. 30, pp. 10513-10518, 2008.

[17] G. Ma, K.-J. Guo, H. Zhang et al., "Expression of programmed cell death 4 and its clinicopathological significance in human pancreatic cancer," Acta Academiae Medicinae Sinicae, vol. 27, no. 5, pp. 597-600, 2005.

[18] Y. Nagao, M. Hisaoka, A. Matsuyama et al., "Association of microRNA-21 expression with its targets, PDCD4 and TIMP3, in pancreatic ductal adenocarcinoma," Modern Pathology, vol. 25, no. 1, pp. 112-121, 2012.

[19] T. Masui, R. Doi, T. Koshiba et al., "RECK expression in pancreatic cancer: its correlation with lower invasiveness and better prognosis," Clinical Cancer Research, vol. 9, no. 5, pp. 1779-1784, 2003. 
[20] F. Orso, F. Balzac, M. Marino, A. Lembo, S. F. Retta, and D. Taverna, "miR-21 coordinates tumor growth and modulates KRIT1 levels," Biochemical and Biophysical Research Communications, vol. 438, no. 1, pp. 90-96, 2013.

[21] H. Yang, W. Kong, L. L. He et al., "MicroRNA expression profiling in human ovarian cancer: miR-214 induces cell survival and cisplatin resistance by targeting PTEN," Cancer Research, vol. 68, no. 2, pp. 425-433, 2008.

[22] J. A. Chan, A. M. Krichevsky, and K. S. Kosik, "MicroRNA-21 is an antiapoptotic factor in human glioblastoma cells," Cancer Research, vol. 65, no. 14, pp. 6029-6033, 2005.

[23] I. A. Asangani, S. A. K. Rasheed, D. A. Nikolova et al., "MicroRNA-21 (miR-21) post-transcriptionally downregulates tumor suppressor Pdcd4 and stimulates invasion, intravasation and metastasis in colorectal cancer," Oncogene, vol. 27, no. 15, pp. 2128-2136, 2008.

[24] L. R. Jiao, A. E. Frampton, J. Jacob et al., "Micrornas targeting oncogenes are down-regulated in pancreatic malignant transformation from benign tumors," PLoS ONE, vol. 7, no. 2, Article ID e32068, 2012.

[25] M. Gironella, M. Seux, M.-J. Xie et al., "Tumor protein 53induced nuclear protein 1 expression is repressed by miR-155, and its restoration inhibits pancreatic tumor development," Proceedings of the National Academy of Sciences of the United States of America, vol. 104, no. 41, pp. 16170-16175, 2007.

[26] C. Huang, H. Li, W. Wu, T. Jiang, and Z. Qiu, "Regulation of miR-155 affects pancreatic cancer cell invasiveness and migration by modulating the STAT3 signaling pathway through SOCS1," Oncology Reports, vol. 30, no. 3, pp. 1223-1230, 2013.

[27] W.-J. Liu, Y.-P. Zhao, T.-P. Zhang et al., "MLH1 as a direct target of MiR-155 and a potential predictor of favorable prognosis in pancreatic cancer," Journal of Gastrointestinal Surgery, vol. 17, no. 8, pp. 1399-1405, 2013.

[28] Q. Liu, J. Chen, J. Wang et al., "Putative tumor suppressor gene SEL1L was downregulated by aberrantly upregulated hsa-mir155 in human pancreatic ductal adenocarcinoma," Molecular Carcinogenesis, vol. 53, no. 9, pp. 711-721, 2014.

[29] N. Habbe, J.-B. M. Koorstra, J. T. Mendell et al., "MicroRNA miR-155 is a biomarker of early pancreatic neoplasia," Cancer Biology and Therapy, vol. 8, no. 4, pp. 340-346, 2009.

[30] J. K. Ryu, S.-M. Hong, C. A. Karikari, R. H. Hruban, M. G. Goggins, and A. Maitra, "Aberrant microRNA-155 expression is an early event in the multistep progression of pancreatic adenocarcinoma," Pancreatology, vol. 10, no. 1, pp. 66-73, 2010.

[31] M. Hackl, S. Brunner, K. Fortschegger et al., "miR-17, miR-19b, miR-20a, and miR-106a are down-regulated in human aging," Aging Cell, vol. 9, no. 2, pp. 291-296, 2010.

[32] H.-L. Yan, G. Xue, Q. Mei et al., "Repression of the miR-17-92 cluster by 533 has an important function in hypoxia-induced apoptosis," The EMBO Journal, vol. 28, no. 18, pp. 2719-2732, 2009.

[33] H. Cai, Y. Yuan, Y.-F. Hao, T.-K. Guo, X. Wei, and Y.-M. Zhang, "Plasma microRNAs serve as novel potential biomarkers for early detection of gastric cancer," Medical Oncology, vol. 30, article 452, 7 pages, 2013.

[34] M. Wang, H. Gu, H. Qian et al., "MiR-17-5p/20a are important markers for gastric cancer and murine double minute 2 participates in their functional regulation," European Journal of Cancer, vol. 49, no. 8, pp. 2010-2021, 2013.

[35] X. Li, J.-H. Pan, B. Song et al., "Suppression of CX43 expression by miR-20a in the progression of human prostate cancer," Cancer Biology and Therapy, vol. 13, no. 10, pp. 890-898, 2012.
[36] H.-W. Kang, F. Wang, Q. Wei et al., "miR-20a promotes migration and invasion by regulating TNKS2 in human cervical cancer cells," FEBS Letters, vol. 586, no. 6, pp. 897-904, 2012.

[37] S. Zhao, D.-S. Yao, J.-Y. Chen, and N. Ding, "Aberrant expression of miR-20a and miR-203 in cervical cancer," Asian Pacific Journal of Cancer Prevention, vol. 14, no. 4, pp. 2289-2293, 2013.

[38] X. Zeng, J. Xiang, M. Wu et al., "Circulating miR-17, miR-20a, miR-29c, and miR-223 combined as non-invasive biomarkers in nasopharyngeal carcinoma," PLoS ONE, vol. 7, no. 10, Article ID e46367, 2012.

[39] G. Huang, K. Nishimoto, Z. Zhou, D. Hughes, and E. S. Kleinerman, "miR-20a encoded by the miR-17-92 cluster increases the metastatic potential of osteosarcoma cells by regulating fas expression," Cancer Research, vol. 72, no. 4, pp. 908-916, 2012.

[40] J.-Y. Li, Y. Zhang, W.-H. Zhang, S. Jia, Y. Kang, and X.-Y. Zhu, "Differential distribution of miR-20a and miR-20b may underly metastatic heterogeneity of breast cancers," Asian Pacific Journal of Cancer Prevention, vol. 13, no. 5, pp. 1901-1906, 2012.

[41] A. Bonauer and S. Dimmeler, "The microRNA-17 92 cluster: still a miRacle?" Cell Cycle, vol. 8, no. 23, pp. 3866-3873, 2009.

[42] Z.-W. Zhang, Y. An, and C.-B. Teng, "The roles of miR-17-92 cluster in mammal development and tumorigenesis," Hereditas, vol. 31, no. 11, pp. 1094-1100, 2009.

[43] H. J. Yan, J. X. Wu, W. S. Liu et al., "MicroRNA-20a overexpression inhibited proliferation and metastasis of pancreatic carcinoma cells," Human Gene Therapy, vol. 21, no. 12, pp. 1723$1734,2010$.

[44] Y.-K. Kim, J. Yu, T. S. Han et al., "Functional links between clustered microRNAs: suppression of cell-cycle inhibitors by microRNA clusters in gastric cancer," Nucleic Acids Research, vol. 37, no. 5, pp. 1672-1681, 2009.

[45] Y. Li, W. Q. Tan, T. W. L. Neo et al., "Role of the miR-106b-25 microRNA cluster in hepatocellular carcinoma," Cancer Science, vol. 100, no. 7, pp. 1234-1242, 2009.

[46] X. Xu, Z. Chen, X. Zhao et al., "MicroRNA-25 promotes cell migration and invasion in esophageal squamous cell carcinoma," Biochemical and Biophysical Research Communications, vol. 421, no. 4, pp. 640-645, 2012.

[47] Q. Li, C. Zou, C. Zou et al., "MicroRNA-25 functions as a potential tumor suppressor in colon cancer by targeting Smad7," Cancer Letters, vol. 335, no. 1, pp. 168-174, 2013.

[48] M. Bloomston, W. L. Frankel, F. Petrocca et al., "MicroRNA expression patterns to differentiate pancreatic adenocarcinoma from normal pancreas and chronic pancreatitis," Journal of the American Medical Association, vol. 297, no. 17, pp. 1901-1908, 2007.

[49] A. E. Szafranska, T. S. Davison, J. John et al., "MicroRNA expression alterations are linked to tumorigenesis and nonneoplastic processes in pancreatic ductal adenocarcinoma," Oncogene, vol. 26, no. 30, pp. 4442-4452, 2007.

[50] M. Liu, Y. Du, J. Gao et al., "Aberrant expression mir-196a is associated with abnormal apoptosis, invasion, and proliferation of pancreatic cancer cells," Pancreas, vol. 42, no. 7, pp. 1169-1181, 2013.

[51] Y. Xue, A. N. Abou Tayoun, K. M. Abo et al., "MicroRNAs as diagnostic markers for pancreatic ductal adenocarcinoma and its precursor, pancreatic intraepithelial neoplasm," Cancer Genetics, vol. 206, no. 6, pp. 217-221, 2013.

[52] M. Sun, X.-H. Liu, J.-H. Li et al., "MiR-196a is upregulated in gastric cancer and promotes cell proliferation by downregulating p27 kip1," Molecular Cancer Therapeutics, vol. 11, no. 4, pp. 842-852, 2012. 
[53] X.-H. Liu, K.-H. Lu, K.-M. Wang et al., "MicroRNA-196a promotes non-small cell lung cancer cell proliferation and invasion through targeting HOXA5," BMC Cancer, vol. 12, article 348, 2012.

[54] M. Eilertsen, S. Andersen, S. Al-Saad et al., "Positive prognostic impact of miR-210 in non-small cell lung cancer," Lung Cancer, vol. 83, no. 2, pp. 272-278, 2014.

[55] R. I. McCormick, C. Blick, J. Ragoussis et al., "MiR-210 is a target of hypoxia-inducible factors 1 and 2 in renal cancer, regulates ISCU and correlates with good prognosis," British Journal of Cancer, vol. 108, no. 5, pp. 1133-1142, 2013.

[56] T. Greither, P. Würl, L. Grochola et al., "Expression of microRNA 210 associates with poor survival and age of tumor onset of soft-tissue sarcoma patients," International Journal of Cancer, vol. 130, no. 5, pp. 1230-1235, 2012.

[57] T. Takikawa, A. Masamune, S. Hamada, E. Nakano, N. Yoshi$\mathrm{da}$, and T. Shimosegawa, "MiR-210 regulates the interaction between pancreatic cancer cells and stellate cells," Biochemical and Biophysical Research Communications, vol. 437, no. 3, pp. 433-439, 2013.

[58] R. K. Mutharasan, V. Nagpal, Y. Ichikawa, and H. Ardehali, "microRNA-210 is upregulated in hypoxic cardiomyocytes through Akt- and p53-dependent pathways and exerts cytoprotective effects," American Journal of Physiology-Heart and Circulatory Physiology, vol. 301, no. 4, pp. H1519-H1530, 2011.

[59] J. H. Kim, S. G. Park, S.-Y. Song, J. K. Kim, and J.-H. Sung, "Reactive oxygen species-responsive miR-210 regulates proliferation and migration of adipose-derived stem cells via PTPN2," Cell Death and Disease, vol. 4, no. 4, article e588, 2013.

[60] A. Giannakakis, R. Sandaltzopoulos, J. Greshock et al., "miR-210 links hypoxia with cell cycle regulation and is deleted in human epithelial ovarian cancer," Cancer Biology and Therapy, vol. 7, no. 2, pp. 255-264, 2008.

[61] S. Tsuchiya, T. Fujiwara, F. Sato et al., "MicroRNA-210 regulates cancer cell proliferation through targeting fibroblast growth factor receptor-like 1 (FGFRL1)," Journal of Biological Chemistry, vol. 286, no. 1, pp. 420-428, 2011.

[62] Y. Yoshioka, N. Kosaka, T. Ochiya, and T. Kato, "Micromanaging iron homeostasis: hypoxia-inducible micro-RNA210 suppresses iron homeostasis-related proteins," Journal of Biological Chemistry, vol. 287, no. 41, pp. 34110-34119, 2012.

[63] H. Wang, S. Bian, and C. S. Yang, "Green tea polyphenol EGCG suppresses lung cancer cell growth through upregulating miR-

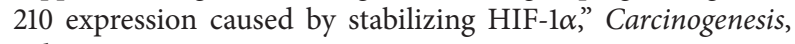
vol. 32, no. 12, pp. 1881-1889, 2011.

[64] X. Huang, L. Ding, K. L. Bennewith et al., "Hypoxia-inducible mir-210 regulates normoxic gene expression involved in tumor initiation," Molecular Cell, vol. 35, no. 6, pp. 856-867, 2009.

[65] C. Camps, F. M. Buffa, S. Colella et al., "Hsa-miR-210 is induced by hypoxia and is an independent prognostic factor in breast cancer," Clinical Cancer Research, vol. 14, no. 5, pp. 1340-1348, 2008.

[66] H. E. Gee, C. Camps, F. M. Buffa et al., "hsa-mir-210 is a marker of tumor hypoxia and a prognostic factor in head and neck cancer," Cancer, vol. 116, no. 9, pp. 2148-2158, 2010.

[67] M. Li, X. Ma, M. Li et al., "Prognostic role of MicroRNA-210 in various carcinomas: a systematic review and meta-analysis," Disease Markers, vol. 2014, Article ID 106197, 10 pages, 2014. 


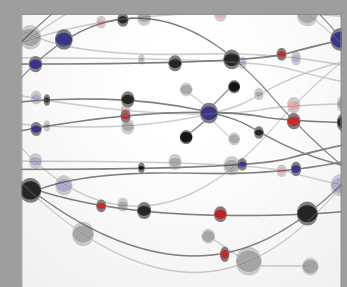

The Scientific World Journal
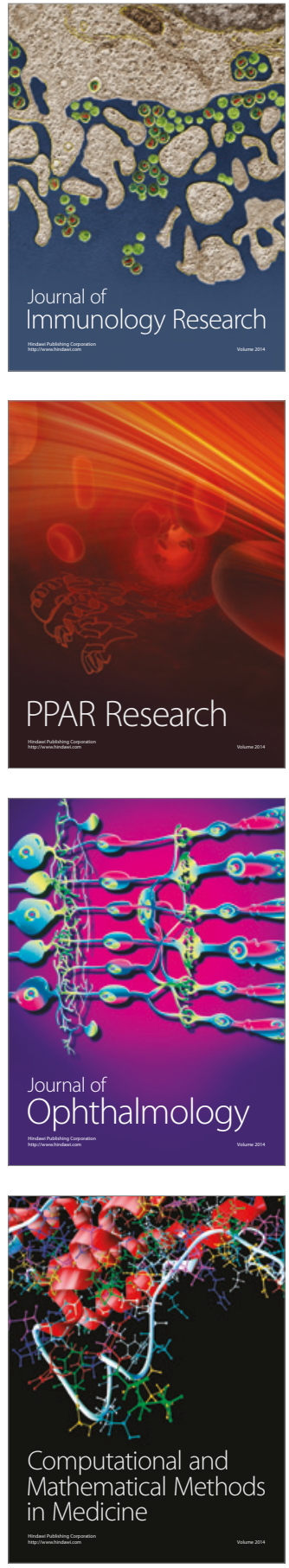

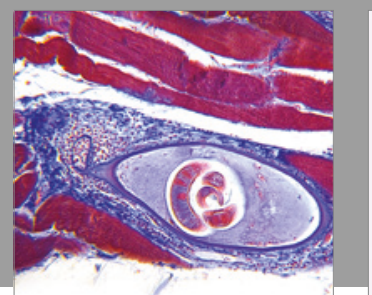

Gastroenterology Research and Practice
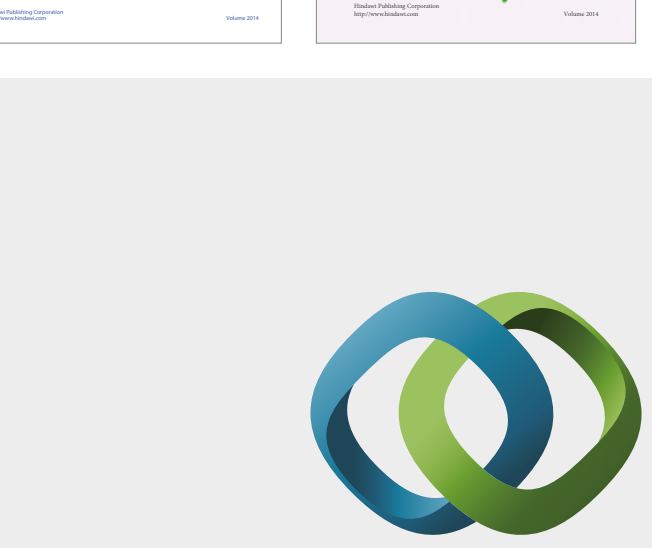

\section{Hindawi}

Submit your manuscripts at

https://www.hindawi.com
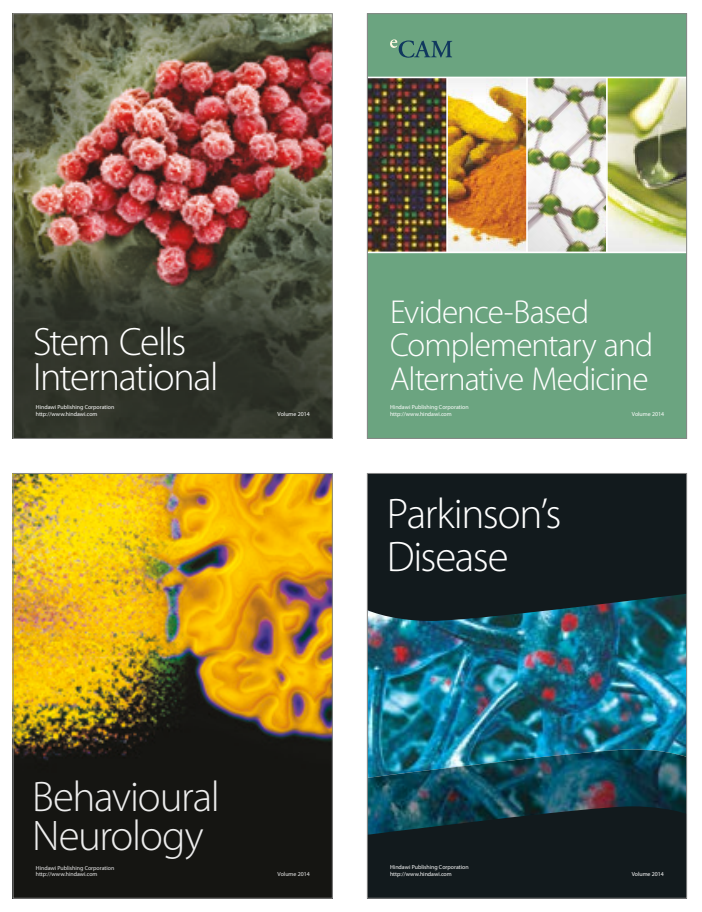
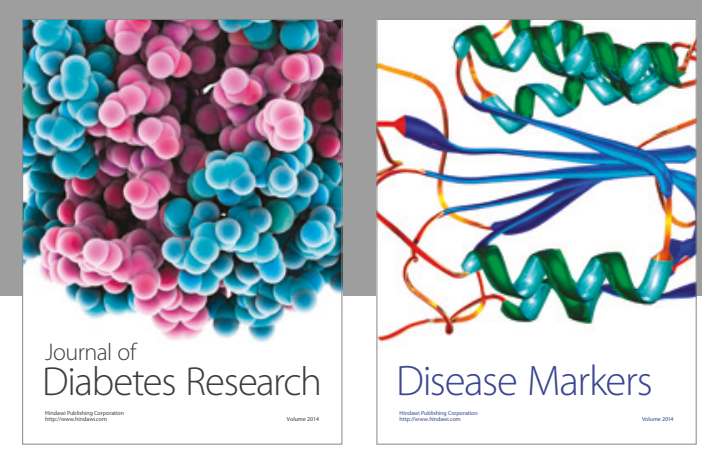

Disease Markers
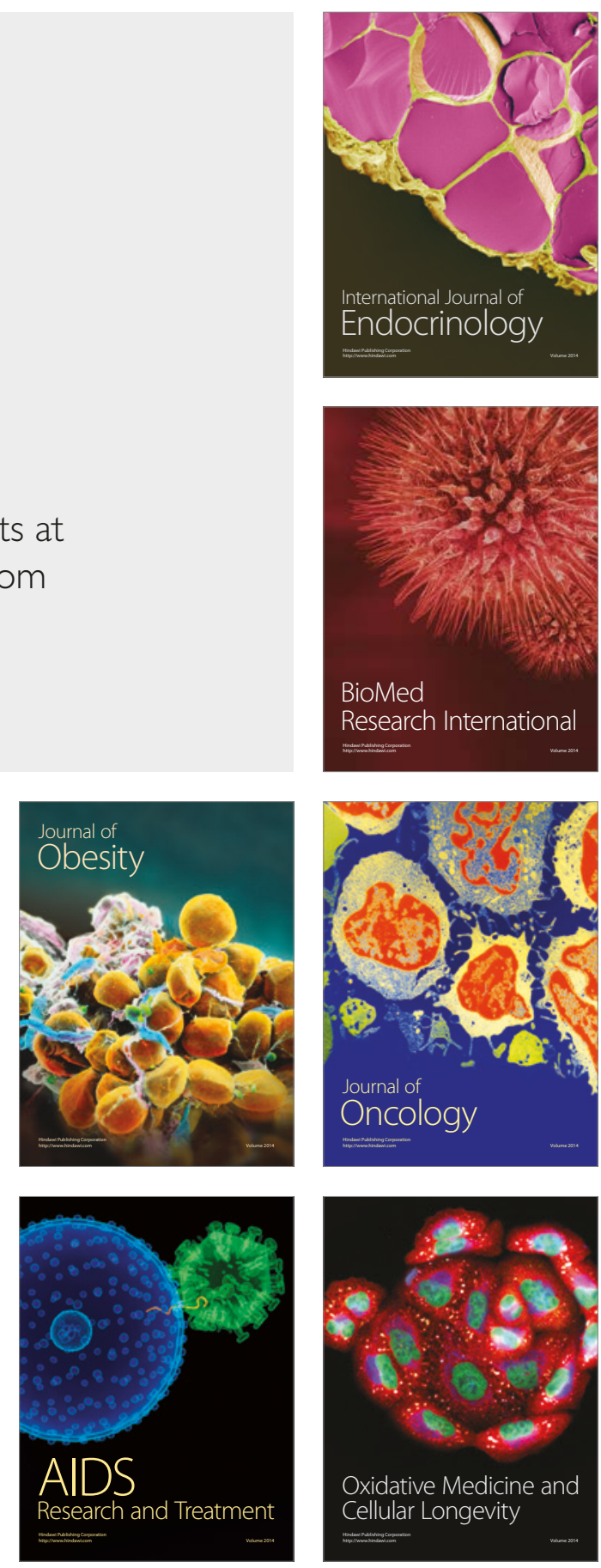